\title{
Differential Effects of Retinoic Acid Concentrations in Regulating Blood-Brain Barrier Properties
}

\author{
Stephanie Bonney and Julie A. Siegenthaler \\ DOI:http://dx.doi.org/10.1523/ENEURO.0378-16.2017 \\ Department of Pediatrics, Section of Developmental Biology School of Medicine Aurora, University of Colorado, CO 80045
}

\begin{abstract}
The blood-brain barrier (BBB) is a multifaceted property of the brain vasculature that protects the brain and maintains homeostasis by tightly regulating the flux of ions, molecules, and cells across the vasculature. Blood vessels in the brain are formed by endothelial cells that acquire barrier properties, such as tight and adherens junctions, soon after the brain vasculature is formed. Endothelial WNT signaling is crucial to induce these BBB properties by regulating their expression and stabilization. Recent studies have implicated retinoic acid (RA) signaling in BBB development and shown that pharmacological concentrations of RA $(\geq 5 \mu \mathrm{m})$ can induce BBB properties in cultured brain endothelial cells. However, a recent study demonstrated that RA inhibits endothelial WNT signaling during brain development, suggesting that RA does not promote BBB properties. We therefore investigated whether RA plays a physiological role in BBB development. We found that BBB function and junctional protein expression was unaffected in mouse mutants that have a reduced capacity to synthesize RA (Rdh10 mutants). Furthermore, embryos exposed to a RA-enriched diet did not enhance BBB protein expression. Together, our data indicate that RA is not capable of inducing, nor is it required for, BBB protein expression in vivo. Like other studies, we found that pharmacological concentrations of RA induce BBB genes in cultured murine brain endothelial cells, and this may involve activation of the LXR/RXR signaling pathway. Our data do not support a role for RA in BBB development, but confirm reports that pharmacological RA is a robust tool to induce BBB properties in culture.
\end{abstract}

Key words: blood brain barrier; brain vascular development; LXR; RXR; endothelial cell

\section{Significance Statement}

Uncovering signals that promote BBB properties in CNS blood vessels is crucial to understand how the brain vascular network supports brain function. In contrast to previous studies, we provide substantial evidence that RA signaling is not required for prenatal BBB development. However, we show that RA at pharmacological concentrations $(\geq 5 \mu \mathrm{M})$ is a useful tool to promote BBB properties in brain ECs and that this effect may be due to LXR/RXR signaling. We have also revealed a potential independent function of LXRs in regulating the expression of BBB genes. These studies could provide insight into mechanisms that underlie the BBB breakdown that occurs in many CNS diseases and improve in vitro BBB models to study drug delivery and BBB biology.

\section{Introduction}

Endothelial cells (ECs) that form blood vessels are specialized in the brain to tightly regulate the transportation of molecules, ions, and cells into and out of the CNS. Various properties are acquired by the brain ECs to form the

Received December 19, 2016; accepted May 12, 2017; First published May 16, 2017.

The authors declare no competing financial interests. blood-brain barrier (BBB), the main being an enrichment of tight junctions (TJs) and adherens junctions (AJs). TJs and AJs work together to seal and strengthen the intercellular connections between brain ECs to form a continuous endothelium. The integrity of these junctional 
complexes is crucial to provide a permissive environment for neuronal function by regulating ionic homeostasis and allowing for effective neuronal action potentials. Furthermore, TJs limit the infiltration of neurotoxic compounds, pathogens, and immune cells, thus preventing CNS injury and disease. Disruption of BBB junctions can affect oxygen and nutrient supply, disrupt ionic homeostasis, and accelerate immune invasion into the CNS. The BBB is also a major obstacle for drug delivery and proper treatment of various neurologic disorders (Hawkins and Davis 2005; Obermeier et al. 2013; Bauer et al. 2014; Engelhardt and Liebner 2014). Therefore, many studies have focused on developing in vitro BBB models to identify drugs that can effectively pass the BBB. However, the molecular cues that promote BBB properties are not fully understood.

CNS ECs obtain BBB properties soon after the blood vessels establish an immature vascular network. In mice, these properties are acquired at approximately embryonic day 13 (E13; Obermeier et al. 2013; Siegenthaler et al. 2013). Although both VEGF and WNT signaling promote angiogenesis of the CNS vasculature, WNT signaling is required for BBB development and maintenance. The WNT transcriptional effector, $\beta$-catenin, regulates the expression of TJ proteins Claudin-3 and Claudin- 5 in brain ECs. Additionally, $\beta$-catenin is required for stabilization of AJs by interacting with VE-cadherin (Liebner et al. 2008; Zhou and Nathans 2014; Zhou et al. 2014).

Recent studies have emerged suggesting a possible role for retinoic acid (RA) signaling in the development of the BBB (Mizee et al. 2013). RA is synthesized from Vitamin $A$ through a series of enzymatic steps that ultimately yield all-trans RA (atRA) and 9-cis RA (9cRA). AtRA binds and activates nuclear receptors called retinoic acid receptors (RARs) with high affinity, whereas 9cRA has high affinity for the retinoid $X$ receptors (RXRs; Tanoury et al. 2013). Mizee et al. (2013) identified astrocytes as a potential source of RA that can induce BBB properties in human brain ECs in vitro. They found that embryos treated with an RAR inhibitor during development displayed increased BBB permeability and reduced vascular expression of the TJ protein ZO-1. Furthermore, these and other studies showed that pharmacological concentrations of atRA $(\geq 5 \mu \mathrm{M})$ can induce TJ and AJ protein expression in both human brain ECs (Mizee et al. 2013) and induced pluripotent stem cell (iPSC)-derived human brain ECs (Lippmann et al. 2014; Katt et al. 2016). Mizee et al. (2013) based their conclusion that RA plays a physiologic role in BBB development in part on the observation that pharmacological concentrations of RA induced BBB gene expression in vitro. The pharmacological concentra-

Institute of Neurological Disorders and Stroke (K99-R00 NS070920 and R01 NS098273 to J.A.S.) and the Children's Hospital of Colorado Research Foundation (start-up funds to J.A.S.).

Correspondence should be addressed to Julie A. Siegenthaler, 12800 E 19th Avenue MS-8313, Aurora, CO 80045. E-mail: Julie.Siegenthaler@ucdenver.edu. DOl:http://dx.doi.org/10.1523/ENEURO.0378-16.2017

Copyright (c) 2017 Bonney and Siegenthaler

This is an open-access article distributed under the terms of the Creative Commons Attribution 4.0 International license, which permits unrestricted use, distribution and reproduction in any medium provided that the original work is properly attributed. tions of atRA used in these studies, while eliciting an effect on BBB properties, are well above what is considered the physiologic range of RA, which is $\sim 2-600 \mathrm{nM}$ (Napoli et al. 1991). Therefore, it is unclear whether RA plays a physiologic role in BBB development. Furthermore, a recent study showed that RA inhibits endothelial WNT signaling during brain vascular development (Bonney et al. 2016), suggesting that RA could limit BBB properties. To understand these discrepancies, we used Rdh10 mutants, which have a reduced capacity to synthesize RA, to investigate a physiologic role for RA in BBB development. We observed that BBB function and junctional protein expression are retained in Rdh10 mutants. Moreover, embryos from pregnant females exposed to an RA-enriched diet did not display enhanced BBB features. Instead, we found a reduction in BBB protein expression; however, BBB leakage was not observed, suggesting that RA exposure does not overtly affect barrier function. Collectively, these data do not support a physiologic role for RA in BBB development. Conversely, we found that pharmacological concentrations of RA induced BBB gene expression in murine brain endothelioma cells, and this effect may be working through complex signaling events that involve LXR/RXR activity.

\section{Methods}

\section{Animals and RA-enriched diets}

Mice used for experiments were housed in specific pathogen-free facilities approved by the American Association for Laboratory Animal Care and were handled in accordance with protocols approved by the animal care committee regulations at the University of Colorado, Denver. The Rdh10 ENU point mutation mutant allele has been described previously (Ashique et al. 2012), and these animals were obtained from Andy Peterson at Genentech. RA-enriched diet (final concentration $0.175 \mathrm{mg} / \mathrm{g}$ food) consisted of atRA (Sigma-Aldrich) dissolved in corn oil and mixed with Bioserv Nutra-Gel Diet, Grain-Based Formula, Cherry Flavor. atRA diet was prepared fresh daily and provided ad libitum to pregnant wild-type females beginning in the afternoon of E10 through the day of collection on E16.

\section{Immunohistochemistry}

Rdh10 mutants (E13.5-E14.5; $n=3$ ), RA-exposed embryos $(\mathrm{E} 16.5 ; n=5)$, and their respective controls (E13.5E14.5: $n=3$; E16: $n=5$ ) were collected, and whole heads or brains were fixed overnight in $4 \%$ paraformaldehyde. All tissues were cryoprotected with $20 \%$ sucrose in PBS and subsequently frozen in OCT. Tissue was cryosectioned in $12-\mu \mathrm{m}$ increments. Immunohistochemistry was performed on tissue sections using the following antibodies: rabbit anti-Claudin-3 1:200 (Invitrogen), rabbit antiClaudin-5 1:200 (Abcam), rabbit anti-fibrinogen 1:500 (Abcam), rat-anti PLVAP 1:100 (AbD Serotec), rabbit antiVE-cadherin 1:200 (Abcam), and mouse anti-ZO-1 1:100 (Thermo Fisher Scientific). After incubation with primary antibodies, sections were incubated with appropriate Alexa Fluor-conjugated secondary antibodies (Invitrogen), Alexa Fluor 633-conjugated isolectin-B4 (Invitrogen), and 
DAPI (Invitrogen). For VE-cadherin staining, tissue was immediately frozen in OCT, cryosectioned, and fixed with methanol for 10 min before immunostaining. For Claudin- 5 staining, antigen retrieval was performed on unfixed tissue, and Claudin-5 expression was detected using a tyramide signal amplification kit according to product specifications (Thermo Fisher Scientific). Immunofluorescent (IF) images were captured using a Zeiss 780 LSM confocal microscope, $40 \times$ objective with $2 \times$ optical zoom to reveal junctional organization. Laser percentage and gain settings were always set on the control tissue. Only few instances occurred where settings required slight reductions (Rdh10 mutants for Claudin-3 and VE-cadherin) or increases (atRA-exposed brains for Claudin-5 and VE-cadherin) to obtain a clear signal.

\section{Immunoblots}

Meninges were removed and forebrains (E16.5) from control $(n=8)$ or RA-exposed $(n=10)$ embryos were collected, homogenized, and lysed in TEN buffer [50 mM Tris, pH 7.5, $150 \mathrm{~mm} \mathrm{NaCl,} \mathrm{0.1 \%} \mathrm{NP-40,} 5$ mм EDTA, 1 mM PMSF, and protease inhibitors (Roche)]. Protein concentration was determined using a BCA kit (Pierce). Lysates were combined with $4 \times$ sample buffer ( $300 \mathrm{~mm}$ Tris, $5 \%$ SDS, $50 \%$ glycerol, $0.025 \%$ bromophenol blue, and $250 \mathrm{~mm} \beta$-mercaptoethanol) and protein (experiment 1, 15 $\mu \mathrm{g}$; experiment 2, $50 \mu \mathrm{g}$ ) was run on Protean Tris- $\mathrm{HCl}$ $4 \%-20 \%$ gradient gel (Bio-Rad) then transferred onto nitrocellulose membranes (Bio-Rad) using the Trans-Blot Turbo System (Bio-Rad). Immunoblots were blocked with $5 \%$ nonfat dehydrated milk (NFDM) in Tris-buffered saline (TBS) with $0.1 \%$ Tween (TBS-T) for $1.5 \mathrm{~h}$ then incubated overnight at $4^{\circ} \mathrm{C}$ in $2.5 \%$ NFDM in TBS-T containing primary antibodies for rabbit anti-Claudin-3 1:250 (Invitrogen), rabbit anti-Claudin-5 1:1000 (Abcam), and rabbit anti-VE-cadherin 1:1000 (Abcam). After primary incubation, blots were washed and incubated in the $2.5 \%$ NFDM containing the appropriate horseradish peroxidase-linked secondary antibody (1:5000; Santa Cruz Biotechnology) for $1 \mathrm{~h}$ at room temperature. Clarity ECL substrate (BioRad) and the ChemiDoc MP system (Bio-Rad) were used to visualize immunolabeled protein bands. Blots were stripped with stripping buffer (Restore Plus; Thermo Fisher Scientific) and reprobed with mouse anti- $\beta$-actin (1:2000; Cell Signaling Technology) antibody as a loading control. Densitometry of bands was performed using ImageLab software (Bio-Rad); density values were corrected for loading variations within each blot using the intensity of $\beta$-actin expression.

\section{Cell culture and pharmacological treatments}

The mouse brain endothelioma cell line (bEnd3.1) was obtained from ATCC (CRL-2299). All experiments were performed on cells from passages 3-8 from when they were received (usually shipped from ATCC at passages within the low 20s), and cells were grown in Dulbecco's minimal essential media with $4.5 \mathrm{~g} / \mathrm{L}$ glucose, $1.5 \mathrm{~g} / \mathrm{L}$ sodium bicarbonate, $4 \mathrm{~mm}$ L-glutamine (Invitrogen), 10\% fetal bovine serum (FBS; Invitrogen), and penicillinstreptomycin (100 U/mL; Invitrogen). Cells were plated on collagen-coated (10 $\mu \mathrm{g} / \mathrm{cm}^{2}$; Sigma) 12-well plates (VWR) for quantitative PCR (qPCR) analysis or four-well glass chambered slides (Nunc Lab-Tek) for immunocytochemistry. Once cells reached confluence, they were serumstarved overnight and treated for $24 \mathrm{~h}$ for mRNA expression analysis or $48 \mathrm{~h}$ for immunocytochemistry analysis with vehicle (DMSO), atRA, or 9cRA (50 nM, $1 \mu \mathrm{M}$, or $5 \mu \mathrm{M}$; Sigma-Aldrich) in serum-free media. To modulate LXR/RXR signaling in vitro, bEnd.3 cultures were treated with vehicle (DMSO), 100 nM GSK-2033 (LXR antagonist; Tocris) with and without $5 \mu \mathrm{M}$ atRA, or $1 \mu \mathrm{M}$ T0901317 (LXR agonist; Tocris) in serum-free DMEM for $24 \mathrm{~h}$ and RNA purified for QPCR and transcriptional analysis. Each treatment condition included three wells of bEnd. 3 cells per experiment. Each experiment was repeated three times $(n=3)$ on two separate cryobatches. Data presented in the main figures are representative of one experiment.

\section{qPCR and expressional analysis}

Meninges were removed from the nonneocortical brain regions of E14 wild-type or Rdh10 mutants $(n=5)$, and RNA was isolated using the RNeasy Mini Kit (Qiagen). Similarly, RNA was isolated and GPCR was performed from cultured bEnd. 3 cells after $24 \mathrm{~h}$ of treatment with DMSO, atRA, 9cRA, GSK-2033, or T0901317. To synthesize cDNA, specifications were followed using the iScript cDNA Synthesis Kit (Bio-Rad) with $1 \mu \mathrm{g}$ of RNA from each sample. To assess transcript levels of Cldn3, Cldn5, Cdh5, and Tjp1 in the tissue samples and Cldn5, Cdh5, Tjp1, Rarb, Gpihbp1, Fabp4, Abcg1, and Apoe in the cell culture experiments, qRT-PCR was performed according to the SYBR Green (Bio-Rad) protocol using the Bio-Rad CFX96 Real Time PCR Detection System. For an internal control, Actb transcript levels were also assessed. To identify expressional differences in control and mutant genotypes or vehicle and treated samples, delta- $\delta$ CT analysis was applied. Primer sequences $\left(5^{\prime}\right.$ to $\left.3^{\prime}\right)$ are as follows: Abcg1 forward: TCCATCGTCTGTACCATCCA, Abcg1 reverse: TACTCCCCTGATGCCACTTC; Actb forward: CTAGGCACCAGGGTGTGAT, Actb reverse: TGCCAGATCTTCTCCATGTC; Apoe forward: AACAGACCCAGCAAATACGC, Apoe reverse: ATGGATGTTGTTGCAGGACA; Cdh5 forward: CAACTTCACCCTCATAAACAACCAT, Cdh5 reverse: ACTTGGCATGCTCCCGAT; Cldn3 forward: CAGACCGTACCGTCACCACT, Cldn3 reverse: ATTCGGCTTGGACAGTTCCT; Cldn5 forward: GCTCTCAGAGTCCGTTGACC, Cldn5 reverse: ATCTAGTGCCCCCAGGATCT; Fabp4 forward: TGTGATGCCTTTGTGGGAAC, Fabp 4 reverse: CGCCCAGTTTGAAGGAAATC; Gpihbp1 forward: CCAGCCCATCATCAAGACAG, Gpihbp1 reverse: GATGAGCAGCCTTGACAACC; Rarb forward: CCAGGAAACCTTTCCCTCAC, Rarb reverse: GAGCAGGGTGATCTGGTCTG; and Tjp1 forward: GCCCTAAACCTGTCCCTCAG, Tjp1 reverse: GCAGAAGGCTTGCTCTCAAA.

\section{Immunocytochemistry}

bEnd.3 cultures were plated on collagen-coated (10 $\mu \mathrm{g} / \mathrm{cm}^{2}$; Sigma-Aldrich) 12-well glass chambered slides (Nunc Lab-Tek) and treated with atRA or 9cRA ( $n=3$; DMSO, $50 \mathrm{nM}, 1 \mu \mathrm{M}$, or $5 \mu \mathrm{M}$ ) in serum-free media for 48 
h after they reached $\sim 80 \%$ confluence and were serumstarved overnight. The cultures were then washed with PBS and fixed with methanol for $10 \mathrm{~min}$ and immunolabeled with rabbit anti-VE-cadherin or mouse anti-ZO-1 (as above). After incubation with primary antibodies, samples were incubated with appropriate Alexa Fluor-conjugated secondary antibodies (Invitrogen) and DAPI (Invitrogen). IF images were captured using a Zeiss 780 LSM confocal microscope using methods as described above. Fluorescent intensity was determined using ImageJ $(\mathrm{NIH})$ and normalized to the number of cells per $20 \times$ image.

\section{Statistics}

Student $t$ tests (parametric) and outlier tests (Grubbs' test) were used to compare the differences between mean values of control (e.g., WT or vehicle) and experimental samples (e.g., Rdh10 mutant, single RA concentration) and to identify significant outliers within data sets, respectively (GraphPad; qPCR analysis, and fluorescence intensity). Student $t$ tests with D'Agostino and Pearson normality (parametric) test were used to compare the differences of BBB protein expression between control and atRA treatment conditions from both experiments. To compare multiple mean values of different treatment conditions (Fig. 5B) to one another, ANOVA (parametric) was used followed by Tukey's test. $p<0.05$ was considered statistically significant. SD is reported on all graphs.

\section{Results}

\section{BBB function and protein expression are not affected in the nonneocortical vasculature of Rdh10 mutants}

In vitro $\mathrm{BBB}$ models have implicated RA signaling in promoting the expression of BBB proteins such as VEcadherin, ZO-1, and Occludin (Mizee et al. 2013; Lippmann et al. 2014; Katt et al. 2016). However, it is unclear whether RA is required for BBB development. To test this, we assessed BBB protein expression in the brains of Rdh10 mutant embryos. Rdh10 mutants survive until E14.5 and have an ENU-induced point mutation in the RA-biosynthetic enzyme Rdh10, resulting in a diminished capacity to synthesize RA (Ashique et al. 2012), thus allowing us to assess whether RA is required for BBB development. Recent work identified defects in blood vessel growth specifically within the neocortices of Rdh10 mutants. This phenotype was attributed to a disruption in vascular WNT signaling. In contrast, the vasculature in the nonneocortical regions (striatum and thalamus) of Rdh10 mutants is relatively normal (Bonney et al. 2016); thus, we investigated the role of RA in BBB protein expression in these regions of wild-type and Rdh10 mutant brains. We examined vascular Claudin-3, Claudin-5, VE-cadherin, and ZO-1 expression via immunohistochemistry (IHC). Punctate Claudin-3 expression, typical of this early developmental stage, was observed in lb4 ${ }^{+}$vessels within the striatum of E13.5-E14.5 control and Rdh10 mutants (Fig. $1 A, B$; arrows). At the same embryonic stages, Claudin-5 (Fig. 1C, D; arrows), VE-cadherin (Fig. 1E, F; arrows), and ZO-1 (Fig. 1G, H; arrows) were expressed and properly localized to the junctions in both control and Rdh10 mutant $\mathrm{Ib}^{+}{ }^{+}$vasculature. We then isolated RNA from E14.5 nonneocortical brain regions (striatum, thalamus, midbrain, and hindbrain) of control and Rdh10 mutants and did qPCR analysis for mRNA expression of Cldn3, Cldn5, Cdh5 (VE-cadherin), and Tjp1 (ZO-1). Cldn3 was significantly up-regulated $(p=0.0221$ ) in Rdh10 mutants (Fig. 1). Claudin-3 is a direct transcription target of WNT signaling in brain endothelial cells. Although vascular WNT signaling is attenuated in the neocortical regions of $R d h 10$ mutants, it was found that WNT signaling is significantly increased in the vasculature of nonneocortical brain regions of Rdh10 mutants (Bonney et al. 2016). Thus the increase in Claudin-3 expression in the nonneocortical regions of Rdh10 mutants could be due to elevated vascular WNT signaling. Cdh5 $(p=0.1559)$ and Tjp1 $(p=$ $0.7461)$ gene expression were not significantly altered in Rdh10 mutants (Fig. 1/). On the other hand, Cldn5 transcript expression was significantly decreased $(p=$ 0.0361 ) in Rdh10 samples (Fig. 1/). This result is unexpected, since Claudin-5 is expressed in blood vessels of mutant brain sections (Fig. 1C, D). We next investigated BBB leakage in Rdh10 mutants by performing IHC for fibrinogen, a serum protein that is restricted from the neural tissue by the BBB. We found retention of fibrinogen in the brain vasculature of both wild-type and Rdh10 mutants (Fig. $1 \mathrm{~J}, K$; arrows), indicating that BBB function is not disrupted when RA levels are reduced. These results show that BBB function and junctional protein expression are intact in Rdh10 mutants, indicating that reductions in RA do not disrupt BBB development and therefore RA is not required for embryonic BBB development.

\section{BBB protein expression is altered after in utero atRA exposure}

To test whether RA is capable of inducing BBB properties during development, we used a prenatal RA exposure paradigm in which we fed pregnant female mice an atRA-enriched diet from E10.5 to E16.5. Based on published reports using a similar diet and quantifying atRA and 9cRA levels in the embryo (Mic et al. 2003), our atRA-enriched diet $(0.175 \mathrm{mg} / \mathrm{g}$ atRA) is not predicted to generate detectable levels of 9cRA in the embryo. Thus RAR signaling, not RXR, is activated in these in vivo experiments. We first analyzed BBB protein expression in the CNS blood vessels of the control or atRA-exposed embryos by IHC. At E16.5, Claudin-3, Claudin-5, VEcadherin, and ZO-1 were observed in blood vessels within the striatum (Claudin-3, VE-cadherin, ZO-1) and cerebral cortex (Claudin-5) of embryos from dams on control diets (Fig. 2A, C, E, G; arrows). In these corresponding brain regions, exposure to the atRA-enriched diet resulted in relatively normal $\mathrm{BBB}$ protein expression and junctional organization within the vasculature (Fig. $2 B, D, F, H$; arrows). However, immunoblot analysis on forebrain lysate (which includes the cortex, striatum, and thalamus) from control and atRA-exposed embryos showed a significant reduction in Claudin-3 $(p=0.0177)$, Claudin-5 $(p=$ $0.0002)$, and VE-cadherin $(p<0.0001)$ expression after atRA exposure (Fig. 2l). We did note that experiment 2 displayed differential expression of $\beta$-actin after atRA exposure; however, we can find no literature that atRA 

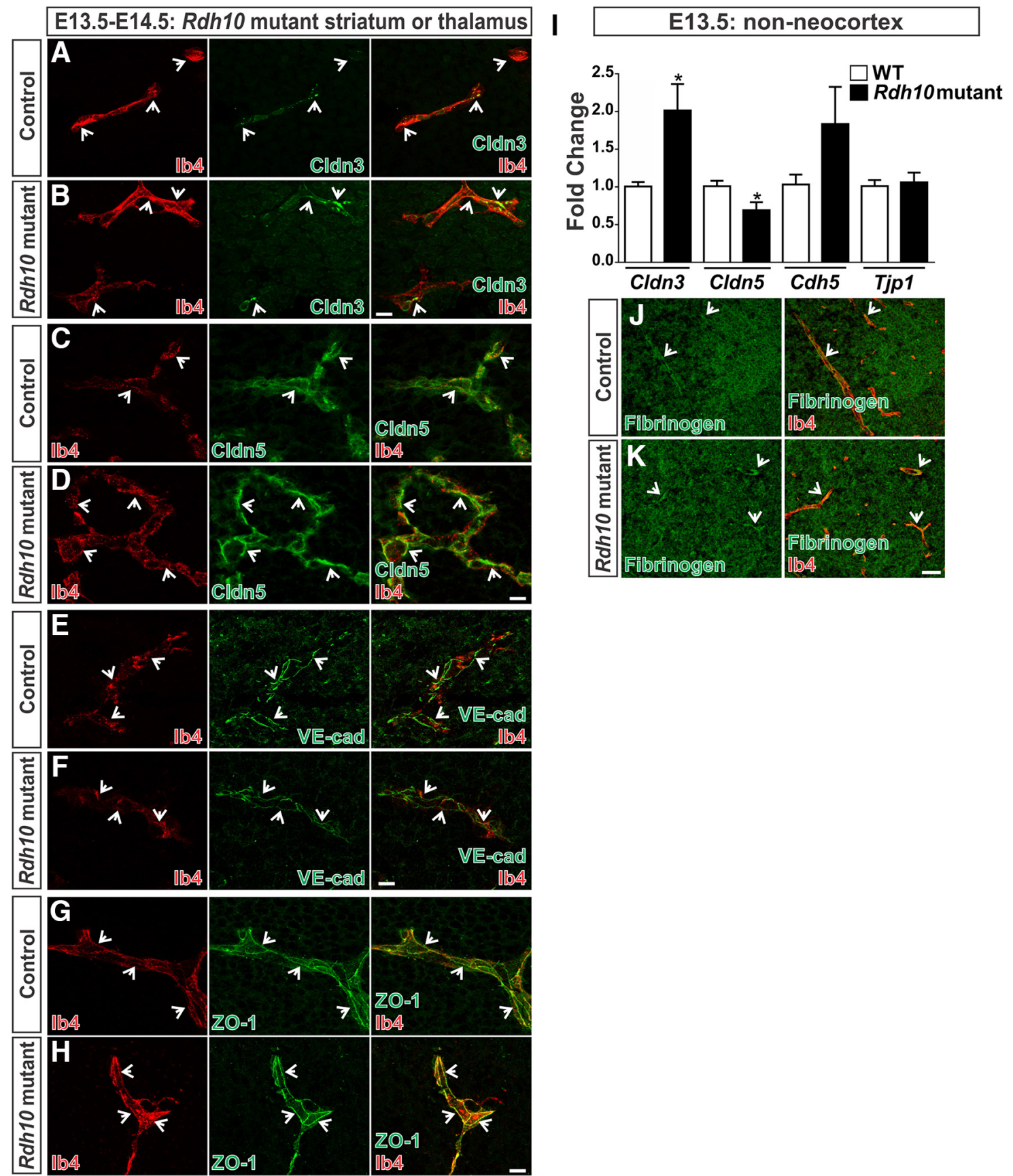

Figure 1. BBB function and protein expression are not affected in the nonneocortical vasculature of $R d h 10$ mutants. $\boldsymbol{A}, \boldsymbol{B}$, E14.5 control $\left(R d h 10^{+/+}\right.$or $\left.R d h 10^{+/-}\right)$and Rdh10 mutant sections immunostained for Claudin-3 (green) and lb4 ${ }^{+}$(red) to visualize blood vessels. Claudin-3 was observed in a punctate pattern in lb4 ${ }^{+}$vessels in the striatum (arrows). $\boldsymbol{C}-\boldsymbol{H}$, Arrows indicate Claudin-5 (C and $\boldsymbol{D}$; green; thalamus), VE-cadherin ( $\boldsymbol{E}$ and $\boldsymbol{F}$; green; thalamus), or ZO-1 ( $\boldsymbol{G}$ and $\boldsymbol{H}$; green; striatum) colocalized with Ib4 ${ }^{+}$vessels (red) and junction formation in both E13.5 control and Rdh10 mutant blood vessels. Scale bars are $10 \mu \mathrm{m}$. I, Graph depicting transcript expression relative to control Actb, as determined by qPCR, for Cldn3, Cldn5, Cdh5 (VE-cadherin), and Tjp1 (ZO-1) in E14.5 control and $R d h 10$ nonneocortices ( $n=5$; striatum, thalamus, midbrain, and hindbrain). Unpaired two-tailed $t$ tests were performed; $*, p<$ 0.05. $\boldsymbol{J}, \boldsymbol{K}, \mathrm{IHC}$ for fibrinogen (green) and $\mathrm{lb}^{+}{ }^{+}$vessels (red) on the striatum of E13.5 mice show retention of fibrinogen within the vasculature of control and Rdh10 mutants (arrows). Scale bar is $50 \mu \mathrm{m}$.

directly modulates $\beta$-actin levels, and this was not observed with atRA treatment in experiment 1.

We next looked for evidence of BBB disruption in the atRA-treated embryos to determine whether the reduction in Claudin-3, Claudin-5, and VE-cadherin expression af- fected BBB integrity. AtRA-exposed embryos showed retention of fibrinogen in the $\mathrm{Ib}^{+}$vasculature (arrows) and no leakage in the neural parenchyma (Fig. $3 A, B$ ). Additionally, we did not observe expression of another indicator of BBB breakdown, PLVAP, in the vasculature in the 


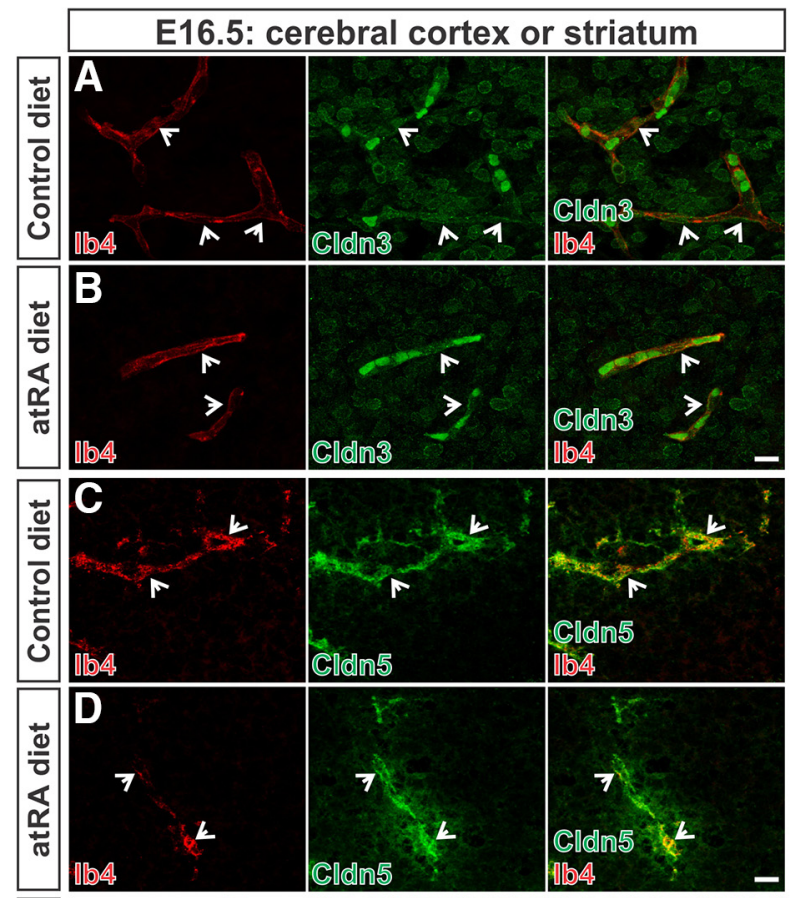

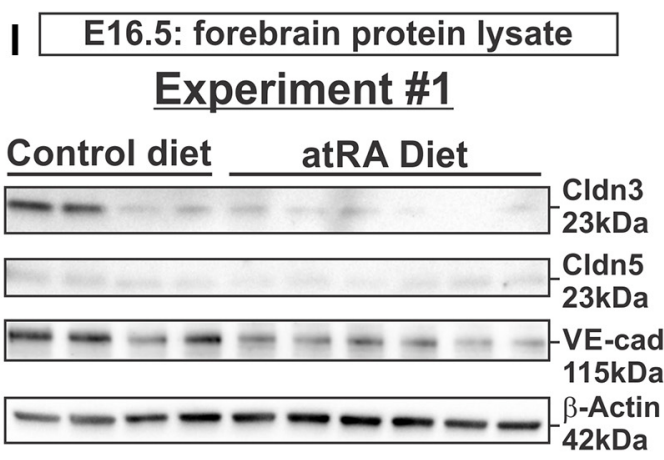

\section{Experiment \#2}
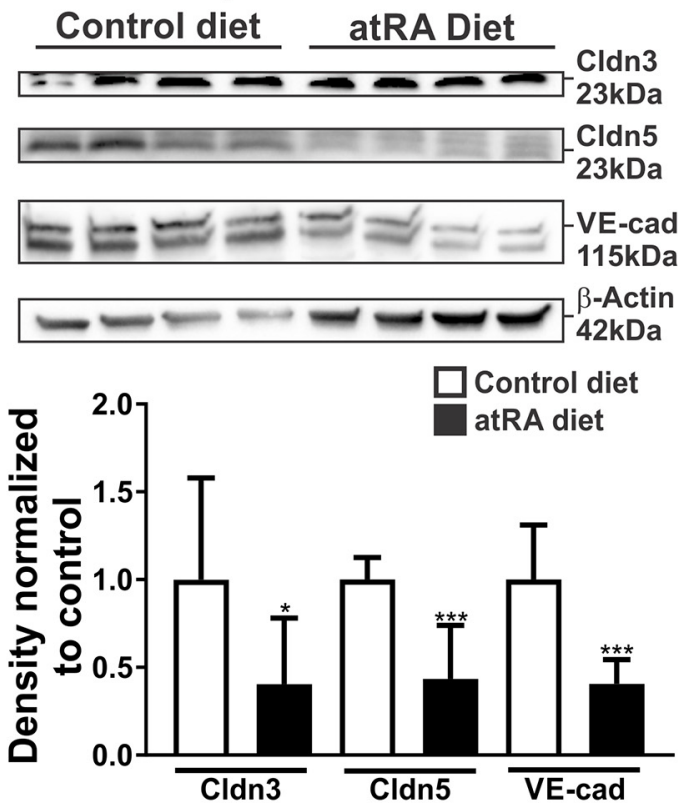

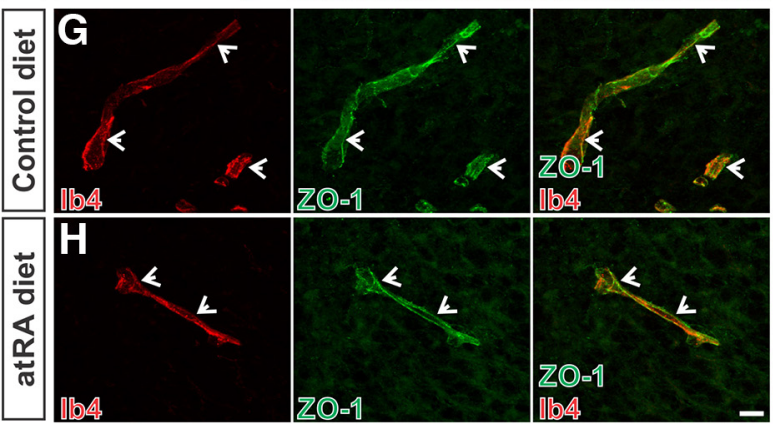

Figure 2. BBB protein expression is altered after in utero atRA exposure. Ib4 ${ }^{+}$blood vessels at the level of the cortex or striatum in E16.5 control and atRA-exposed brains immunolabeled with antibodies to Claudin-3 (A and $\boldsymbol{B}$; green; striatum), Claudin-5 ( $\boldsymbol{C}$ and $\boldsymbol{D}$; green; cortex), VE-cadherin ( $\boldsymbol{E}$ and $\boldsymbol{F}$; green; striatum), or ZO-1 ( $\boldsymbol{G}$ and $\boldsymbol{H}$; green; striatum) and colabeled with Ib4 (red). Arrows indicate positive staining within vasculature for each protein. Junction formation is clear in VE-cadherin and ZO-1 staining. Scale bars are $10 \mu \mathrm{m}$. I, Immunoblots for Claudin-3, Claudin-5, VE-cadherin, and $\beta$-actin were performed on E16.5 forebrain lysates from two different experiments of control or atRA-exposed embryos. Graph depicting densitometry (protein of interest intensity/ $\beta$-actin intensity) and reduced BBB protein expression in atRA-exposed samples $(n=10)$ when normalized to control diet samples $(n=8)$. Full immunoblots are shown in Fig. $2-1$. Unpaired two-tailed $t$ tests were performed; $*, p<0.05$, $* * *, p<0.001$.

atRA-exposed embryos (Fig. 3C, D; arrows). Given these observations, we conclude that atRA exposure does not promote BBB features and instead may reduce BBB protein expression; however, not to the extent that BBB integrity is reduced in the prenatal brain.

\section{Differential effects of RA concentrations on BBB gene expression}

Recent studies showed that pharmacological concentrations of atRA ( 5 and $10 \mu \mathrm{M}$ ) are capable of inducing the expression of TJ and AJ genes (Mizee et al. 2013; Lip- 


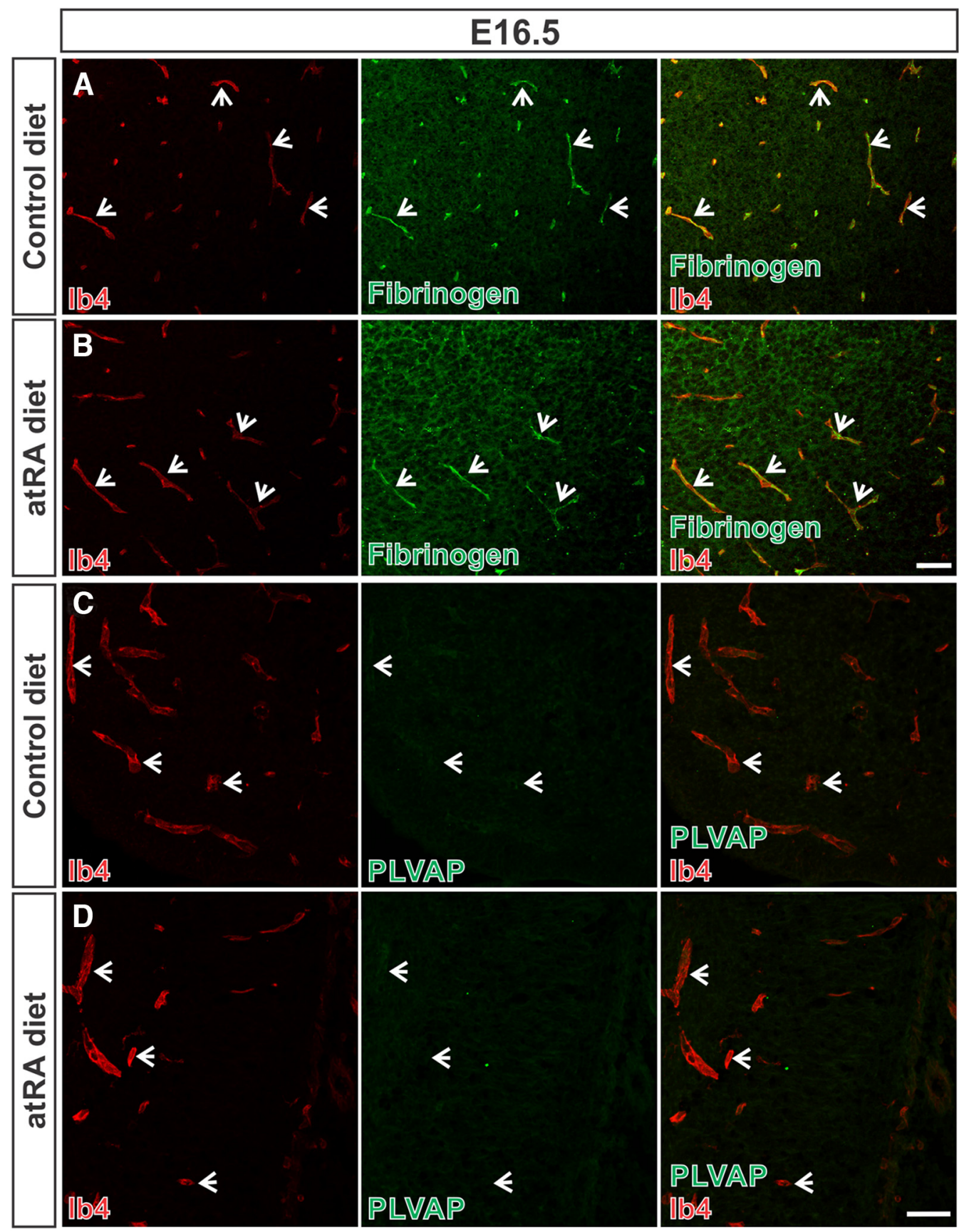

Figure 3. In utero atRA exposure does not overtly affect BBB integrity. $\boldsymbol{A}, \boldsymbol{B}$, Arrows show that fibrinogen (green) is contained with the lumen of thalamic vessels labeled with lb4 (red) and not observed in the parenchyma of control and atRA-exposed E16.5 fetal brains. $\boldsymbol{C}, \boldsymbol{D}$, PLVAP (green; cortex) expression is absent in the lb4-labeled vasculature (arrows) in the control and atRA-exposed E16.5 fetal brains. Scale bars are $50 \mu \mathrm{m}$.

pmann et al. 2014; Katt et al. 2016). Because the physiologic role of RA in BBB protein expression is unclear, we first assessed TJ and AJ gene expression after treatment with $50 \mathrm{~nm}$ atRA, which is within the range of atRA detected in tissues ( 2-600 nM; Napoli et al. 1991), in a murine brain endothelioma cell line (bEnd.3). bEnd.3 cells are a transformed brain endothelial cells from 6-week-old $\mathrm{Balb} / \mathrm{c}$ mice. When used at lower passage numbers $(<30)$, this cell line displays barrier properties, such as permeability and transendothelial electrical resistance, compara- ble to those of primary brain ECs. They also highly express BBB proteins such as Claudin5 and ZO-1. Therefore, these cells are a suitable alternative to primary brain ECs when studying BBB function and drug delivery owing to their rapid growth when used at appropriate passages (Brown et al. 2007; Watanabe et al. 2013). After exposure of $50 \mathrm{~nm}$ atRA in the bEnd.3 cells, we found that the expression of Cldn5 $(p=0.0106)$ and Cdh5 $(p=0.0159)$ was significantly reduced after $24 \mathrm{~h}$; however, Tjp1 ( $p=$ $0.2996)$ expression was not altered (Fig. 4A). This concen- 


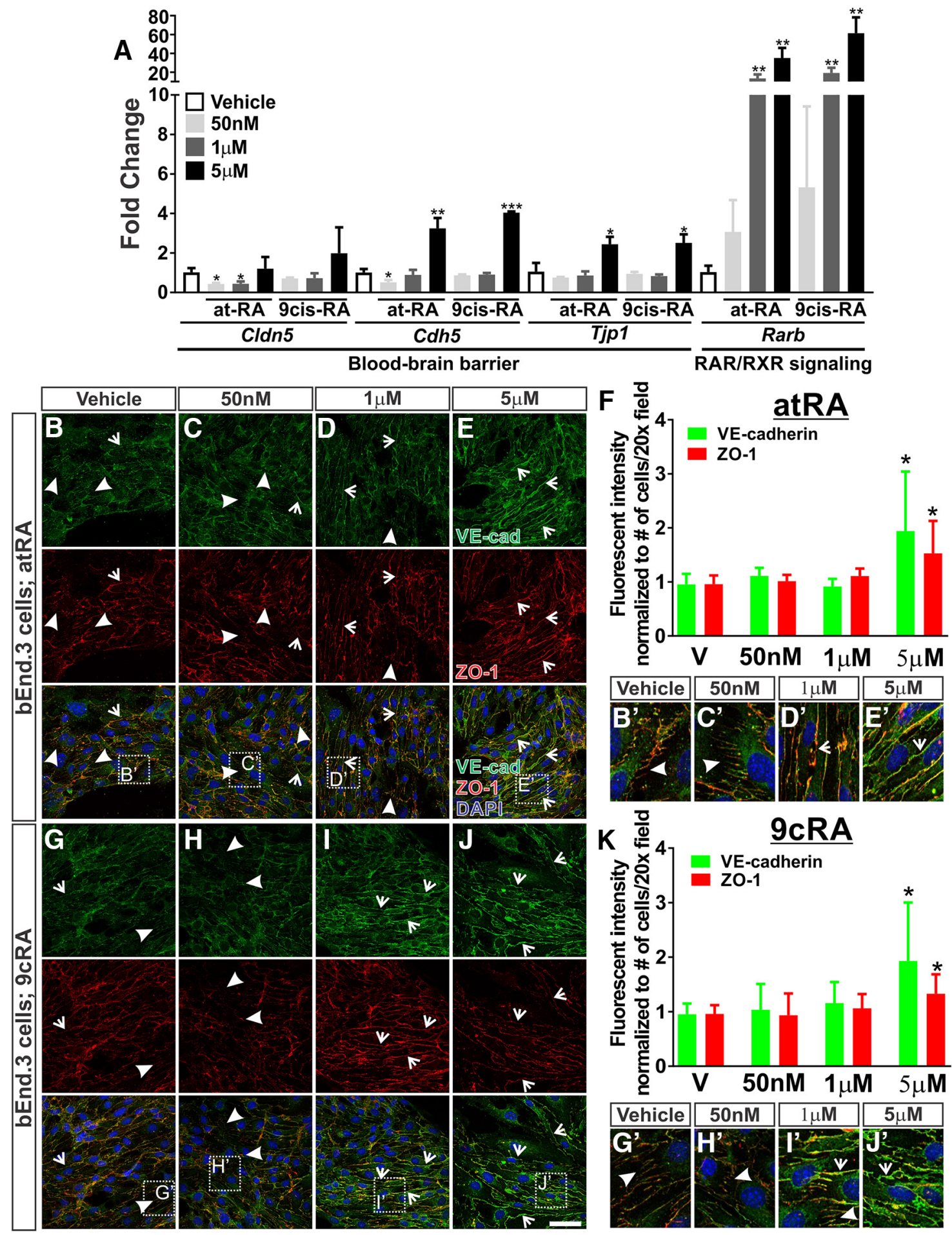

Figure 4. Differential effects of RA concentrations on BBB gene expression. $\boldsymbol{A}$, Representative graph depicting transcript expression of Cldn5, Cdh5, Tjp1, and Rarb normalized to Actb after $24 \mathrm{~h}$ of treatment with DMSO, $50 \mathrm{~nm}, 1 \mu \mathrm{M}$, or $5 \mu \mathrm{M}$ of atRA or 9cRA show differential effects of RA concentrations in bEnd.3 cells (murine brain endothelioma cell line). Trend was consistent between all experiments $(n=3)$. All experiments (1-3) are shown in Fig. 4-1. $\boldsymbol{B}-\boldsymbol{K}$, Immunocytochemistry of bEnd.3 cultures after treatments with atRA (B-F; DMSO, $50 \mathrm{~nm}, 1 \mu \mathrm{M}$, and $5 \mu \mathrm{M})$ and 9cRA (G-K; DMSO, $50 \mathrm{~nm}, 1 \mu \mathrm{M}$ and $5 \mu \mathrm{M}$ ) for VE-cadherin (green), ZO-1 (red), and DAPI (blue) after 48 h. $\boldsymbol{F}, \boldsymbol{K}$, Fluorescence intensity of VE-cadherin (green) and ZO-1 (red) after treatments with atRA (F; DMSO, 50 $\mathrm{nm}, 1 \mu \mathrm{M}$, and $5 \mu \mathrm{M})$ or $9 \mathrm{cRA}(\boldsymbol{K}$; DMSO, $50 \mathrm{nM}, 1 \mu \mathrm{M}$, and $5 \mu \mathrm{M}) . \boldsymbol{B}^{\prime}-\boldsymbol{E}^{\prime}, \boldsymbol{G}^{\prime}-\boldsymbol{J}^{\prime}$, Enlarged images highlight junctional organization after treatments with atRA $\left(\boldsymbol{B}^{\prime}:\right.$ DMSO; $\left.\boldsymbol{C}^{\prime}: 50 \mathrm{~nm} ; \boldsymbol{D}^{\prime}: 1 \mu \mathrm{M} ; \boldsymbol{E}^{\prime}: 5 \mu \mathrm{M}\right)$ and 9cRA (G': DMSO; $\left.\boldsymbol{H}^{\prime}: 50 \mathrm{~nm} ; \boldsymbol{I}^{\prime}: 1 \mu \mathrm{m} ; \boldsymbol{J}^{\prime}: 5 \mu \mathrm{M}\right)$. Arrowheads show occurrence of disjointed and disorganized junctions in physiologic concentrations of atRA and 9cRA. Arrows indicate improved junction formation in pharmacological concentrations of atRA and 9cRA. Unpaired two-tailed $t$ tests were performed, $*, p<0.05$, $* *, p<0.01, * * *, p<0.001$. Scale bars are $50 \mu \mathrm{m}$. 
tration of atRA was shown to inhibit WNT transcriptional activity (Bonney et al. 2016). Therefore, this effect may occur through RA-mediated suppression of endothelial WNT signaling. We next tested a high pharmacological concentration of atRA $(5 \mu \mathrm{M})$ that has been previously shown to increase BBB gene and protein expression (Mizee et al. 2013; Lippmann et al. 2014; Katt et al. 2016). We observed that $5 \mu \mathrm{M}$ atRA up-regulated Cdh5 $(p=$ 0.0022 ) and Tjp1 ( $p=0.0138$; Fig. $4 A$ ). Cldn5 expression was not significantly altered $(p=0.6336)$ after $5 \mu \mathrm{M}$ atRA exposure (Fig. 4A). An intermediate concentration of atRA $(1 \mu \mathrm{M})$ did not alter Cdh5 $(p=0.5286)$ or Tjp1 $(p=0.5246)$ expression; however, Cldn5 was significantly reduced $(p=0.0154$; Fig. 4A). The increase in Tjp 1 and Cdh5 transcript expression after the pharmacological RA treatments $(5 \mu \mathrm{M})$ in our studies using the bEnd. 3 cell line follows a trend similar to that of studies using human brain ECs (Mizee et al. 2013) and iPSC-derived brain ECs (Lippmann et al. 2014; Katt et al. 2016).

High concentrations of atRA have been shown to isomerize to 9cRA (Urbach and Rando 1994); therefore, the high atRA concentrations may act via 9cRA. We tested whether 9cRA is able to regulate BBB gene expression and, interestingly, $50 \mathrm{~nm}$ 9cRA had little effect on Cldn5 ( $p=0.2003)$, Cdh5 $(p=0.3880)$, and Tjp1 $(p=$ $0.4259)$. However the pharmacological concentrations of 9cRA, similar to the effect of atRA, induced Cdh5 $(p=$ $0.0002)$ and Tjp1 $(p=0.0349)$ but not Cldn5 $(p=0.2750$; Fig. 4A). Although the BBB genes showed differential responses to the RA concentrations, Rarb, a RAR/RXR target gene, was elevated at all concentrations with both atRA and 9cRA, with the exception of $50 \mathrm{~nm}$ atRA and 9cRA. However, all experiments performed showed consistent elevation of Rarb at all concentrations (atRA: 50 $\mathrm{nM}, p=0.1003 ; 1 \mu \mathrm{M}, p=0.0078 ; 5 \mu \mathrm{M}, p=0.0051$; 9cRA: $50 \mathrm{nM}, p=0.1451 ; 1 \mu \mathrm{M}, p=0.0044 ; 5 \mu \mathrm{M}, p=$ 0.0065; Fig. 4A) This indicates that RAR and RXR transcriptional activity was activated at all concentrations.

We next looked at protein expression and junctional organization of ZO-1 and VE-cadherin by immunocytochemistry in the bEnd. 3 cells after exposure to the physiologic $(50 \mathrm{nM})$ and pharmacological (1 and $5 \mu \mathrm{M}$ ) concentrations of atRA and 9cRA for $48 \mathrm{~h}$. Consistent with our transcript analysis, fluorescent intensity of VEcadherin increased significantly after $5 \mu \mathrm{M}$ atRA ( $p=$ 0.0273 ) and 9cRA ( $p=0.0256$; Fig. $4 E, F, J, K$; green) but was unaltered at lower concentrations of atRA or 9cRA. ZO-1 intensity increased after $5 \mu \mathrm{M}$ atRA $(p=0.0263)$ and 9cRA ( $p=0.0285$; Fig. 4E, $F, J, K$; red); however, other concentrations of atRA or 9cRA did not have a significant effect on ZO-1 protein expression. Enlarged images revealed disjointed and disorganized junctions (as indicated by the VE-cadherin and ZO-1 staining) in the vehicle and 50 nм RA cultures (Fig. 4B', $C^{\prime}, G^{\prime}, H^{\prime}$; arrowheads); however, junctions appear improved with the pharmacological concentrations (Fig $4 D^{\prime}, E^{\prime}, I^{\prime}, J^{\prime}$; arrows), further suggesting an increase in junctional protein expression. These investigations corroborate previous studies showing that pharmacological concentrations of atRA ( $5 \mu \mathrm{M})$ promote BBB properties and suggest a possible role for 9cRA-RXR signaling in regulating BBB gene expression.

\section{Induction of BBB genes by pharmacological RA correlates with activation of LXR/RXR signaling}

Microarray analysis on ECs isolated from various mouse organs have shown that RXR signaling is enriched in CNS ECs (Daneman et al. 2010). Because our investigations suggested a potential role for 9cRA-RXR signaling in regulating $\mathrm{BBB}$ gene expression, we next investigated induction of different pathways that involve RXRs, peroxisome proliferator-activated receptor (PPAR), and liver $X$ receptor (LXR) signaling, by pharmacological concentrations of atRA and 9cRA. Both PPARs and LXRs can heterodimerize with RXRs and regulate gene expression (Szanto et al. 2004). Treatment with the pharmacological concentrations of atRA or 9cRA did not induce two PPAR/ RXR target genes, Gpihbp1 and Fabp4, suggesting that PPAR/RXR signaling is not involved in the induction of the BBB genes (Fig. 5A). However, two LXR/RXR targets, $A b c g 1$ and Apoe, showed expression patterns similar to those of the BBB transcripts after RA treatment, where they were down-regulated with the physiologic RA concentrations (50 nм; atRA: Apoe, $p=0.0176$; Abcg1, $p=$ 0.0039; 9cRA: Apoe, $p=0.0312$; Abcg1, $p=0.0097$ ) and up-regulated with the pharmacological concentrations of atRA and 9cRA (5 $\mu \mathrm{M}$; atRA: Apoe, $p=0.0091 ; A b c g 1, p$ $=0.0011$; 9cRA: Apoe, $p=0.0006 ;$ Abcg1, $p=0.0074$; Fig. $5 A$ ). This suggests that pharmacological concentrations of RA can activate the LXR/RXR pathway in culture. Thus we hypothesized that pharmacological concentrations of RA induce the expression of BBB genes through LXR/RXR signaling. To test this, we treated bEnd.3 cultures with $5 \mu \mathrm{M}$ atRA with and without the LXR antagonist, GSK-2033, and assessed the expression of the BBB transcripts. Unexpectedly, the addition of GSK-2033 to the 5 $\mu \mathrm{M}$ atRA-treated cultures significantly enhanced the RAmediated up-regulation of Tjp1 $(p=0.0053)$ compared with the $5 \mu \mathrm{M}$ atRA samples (Fig. $5 B$ ). The expression of Cldn5, which was not induced by $5 \mu \mathrm{M}$ atRA alone (Figs. $4 A$ and $5 B$ ), was significantly up-regulated with $5 \mu \mathrm{M}$ atRA plus GSK-2033 $(p=0.0037)$ compared with vehicletreated samples or $5 \mu \mathrm{M}$ atRA alone ( $p=0.0040$; Fig. $5 B$ ). Cdh5 was significantly elevated in cultures treated with 5 $\mu \mathrm{M}$ atRA plus GSK-2033 compared with vehicle-treated cultures $(p=0.0074)$; however, this was not significant compared with $5 \mu \mathrm{M}$ atRA alone $(p=0.0740$; Fig. $5 B)$. $A b c g 1$ expression was unchanged in cultures exposed to GSK-2033 and $5 \mu \mathrm{M}$ atRA compared with control samples ( $p=0.8535$ ); however, Apoe was significantly upregulated in bEnd. 3 cultures treated with GSK-2033 and 5 $\mu \mathrm{M}$ atRA $(p=0.0041)$. In comparison to vehicle-treated cultures, GSK-2033 did not have a significant effect on Apoe expression ( $p=0.9987$ ); however, Abcg1 was down-regulated $(p=0.0098)$, indicating that GSK-2033 can inhibit LXR signaling (Fig. 5B). Rarb was up-regulated in cultures treated with $5 \mu \mathrm{M}$ atRA $(p=0.0002)$ and $5 \mu \mathrm{M}$ atRA plus GSK-2033 ( $p=0.0001)$ but not with GSK-2033 alone $(p=0.9989)$ compared with vehicle-treated cultures (Fig. 5B). Together, these data suggest that inhibi- 
A
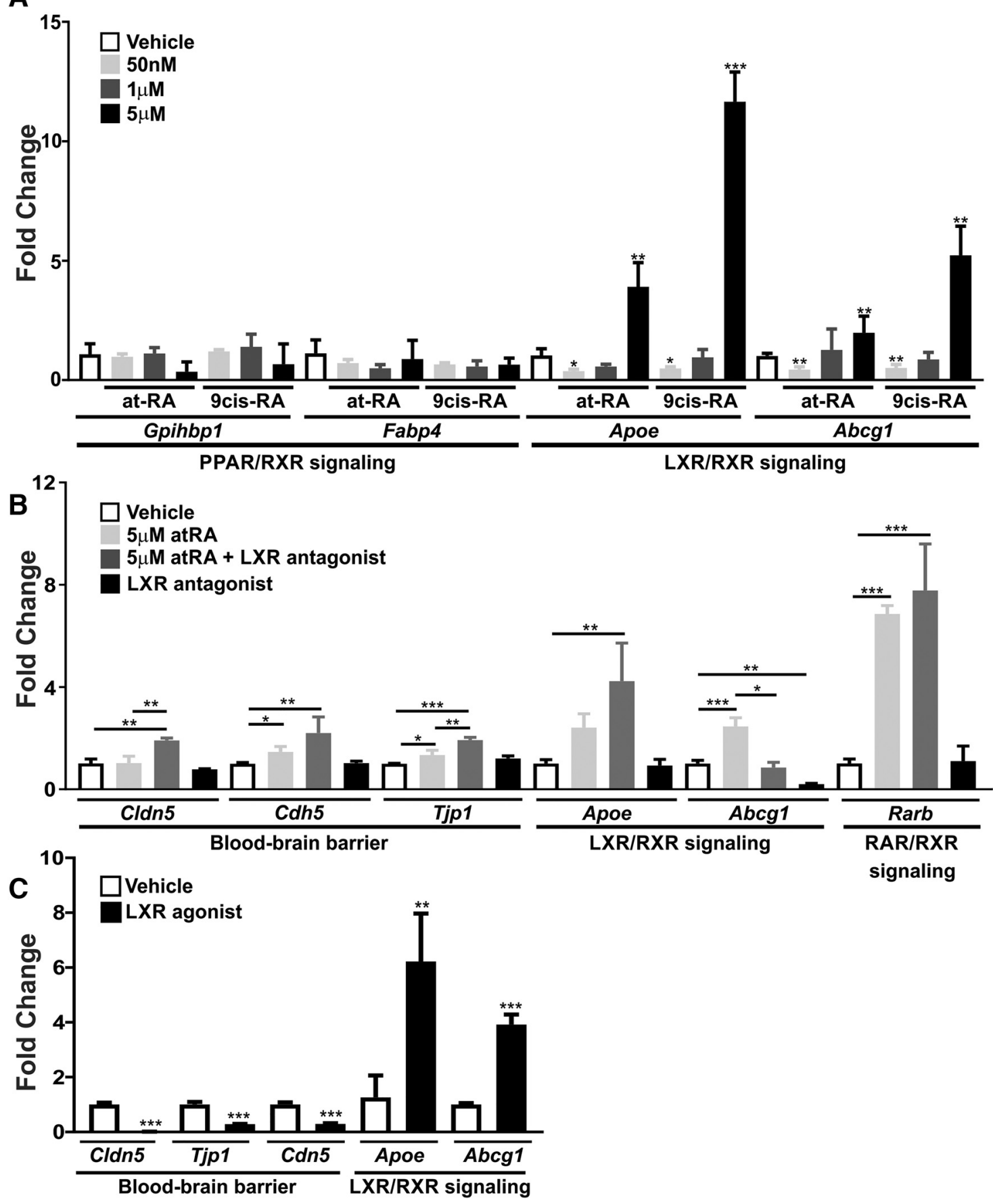

Figure 5. Induction of BBB genes by pharmacological RA correlates with activation of LXR/RXR signaling. $\boldsymbol{A}$, Representative graph depicting transcript expression of PPAR/RXR target genes, Gpihbp1 and Fabp4, and LXR/RXR signaling genes, Apoe and Abcg1, normalized to Actb after $24 \mathrm{~h}$ of treatment with DMSO, $50 \mathrm{nM}, 1 \mu \mathrm{M}$, or $5 \mu \mathrm{M}$ of atRA or $9 \mathrm{cRA}$ in b. End3 cells. Graph depicts induction of LXR/RXR genes following pharmacological concentrations of RA. With the exception of Gpihbp1 and Fabp4, Apoe and Abcg1 expression was consistent between all experiments $(n=3)$. All experiments (1-3) are shown in Fig. 4-1. $\boldsymbol{B}$, Representative graph depicting transcript expression of Cldn5, Cdh5, Tjp1, Apoe, Abcg1, and Rarb normalized to Actb after $24 \mathrm{~h}$ of treatment with DMSO, $5 \mu \mathrm{m}$ atRA, $5 \mu \mathrm{m}$ atRA + $100 \mathrm{~nm}$ GSK-2033 (LXR antagonist), or $100 \mathrm{~nm}$ GSK-2033 in b.End3 cells. Inhibition of LXR signaling enhanced the induction of BBB gene expression by the pharmacological RA concentrations. Trend was consistent between all experiments $(n=3)$. All experiments (1-3) are shown in Fig. 4-1. ANOVA analysis followed by Tukey's test was used to compare the mean values of multiple treatment conditions to one another. C, Representative graph depicting transcript expression of Cldn5, Cdh5, Tjp1, Apoe, and $A b c g 1$ normalized to Actb after $24 \mathrm{~h}$ of treatment with DMSO or $1 \mu \mathrm{M}$ T0901317 (LXR agonist) reveals LXR-mediated inhibition of BBB gene expression in b.End3 cells. Trend was consistent between all experiments $(n=3)$. All experiments $(1-3)$ are shown in Fig. 4-1. Unpaired two-tailed $t$ tests were performed, $*, p<0.05, * *, p<0.01, * * *, p<0.001$ 
tion of LXR activity with GSK-2033 enhances the RAmediated up-regulation of the BBB genes. GSK-2033 was recently shown to augment RXR transcriptional activity (Griffett and Burris 2016); thus, it is possible that the increase in BBB gene expression is due to enhanced RXR transcriptional activation by GSK-2033. However, our data indicate that GSK-2033 alone is not sufficient to induce the expression of BBB genes (Fig. 5B; Cldn5, $p=$ 0.4356; Cdh5, $p=0.9993$; Tjp1, $p=0.2588$ ) potentially because of the indirect mechanism of RXR activation. Additionally, LXRs have been known to suppress gene expression independently of RXRs, a mechanism termed LXR transrepression (Jakobsson et al. 2012). Therefore, it is also possible that GSK-2033 inhibited independent LXR activity and relieved LXR-mediated suppression of BBB gene expression. To test this, we treated bEnd. 3 cells with T0901317, a potent LXR agonist. We found that Cldn5 ( $p$ $<0.0001)$, Cdh5 $(p=0.0002)$, and Tjp1 $(p=0.0001)$ were significantly down-regulated in bEnd.3 cultures exposed to T0901317 (Fig. 5C). Apoe ( $p=0.0013$ ) and Abcg1 $(p=$ $0.0002)$ were significantly up-regulated, verifying activation of LXR signaling using T0901317 (Fig. 5C). This suggests that LXRs independently inhibit BBB gene expression. Collectively, these studies show that pharmacological concentrations of atRA can activate the LXR/ RXR signaling pathway, which may be involved in the regulation of $\mathrm{BBB}$ gene expression observed in brain endothelial cell cultures.

\section{Discussion}

We have investigated the role of RA signaling in the development of the BBB and the signaling pathways activated by RA that regulate BBB gene expression in culture. We conclude that $R A$ is not required for $B B B$ function and the expression of BBB proteins by the developing CNS vasculature. Furthermore, excess RA does not induce BBB protein expression by the developing brain vasculature (Fig. 6A). Although our studies do not point to a physiologic role for RA in promoting BBB properties in the embryonic brain, we found that pharmacological concentrations of atRA $(\geq 5 \mu \mathrm{M})$ up-regulate BBB gene expression and activate both RAR/RXR and $L X R / R X R$ signaling. Although our data indicate that LXR/ $R X R$ signaling could be involved in the regulation of BBB genes, we also identified a potential role for LXR transrepression in regulating BBB properties (Fig. 6B).

RA has been implicated in the maturation of the BBB through regulation of BBB protein expression (Mizee et al. 2013; Lippmann et al. 2014; Katt et al. 2016). A common finding between these studies was elevated VE-cadherin and the TJ protein Occludin after pharmacological treatments of atRA in cultured human ECs. Furthermore, Mizee et al. (2013) showed that VE-cadherin transcript and BBB integrity were decreased in the fetal brain of embryos after maternal exposure to a pan-RAR inhibitor (BMS-493), which inhibits RAR transcriptional activity by promoting interactions with transcriptional corepressors. This systemic treatment, however, would block RAR transcriptional activity in neural cells, pericytes, and ECs. Although Rdh10 mutants have diminished amounts of synthesized RA and therefore reduced RAR signaling in these different cell types, we found that VE-cadherin protein and transcript expression was not significantly altered in nonneocortical brain regions of global RA-deficient Rdh10 mutants. This indicates that RA is not required to induce VE-cadherin in brain ECs during prenatal development. Additionally, embryos exposed to a maternal atRAenriched diet to elevate RA levels had diminished VEcadherin protein expression. This could be a result of decreased brain endothelial WNT signaling as has been observed with prenatal atRA exposure (Bonney et al. 2016). Although VE-cadherin interacts with WNT signaling component, $\beta$-catenin, at AJs, it is not known whether the WNT pathway directly regulates VE-cadherin expression. Alternatively, reductions in VE-cadherin expression could result directly from enhanced endothelial RA signaling. In addition to VE-cadherin, we observed reduced Claudin-3 and Claudin-5 expression, known targets of endothelial WNT signaling, in forebrain lysate of atRA-exposed embryos. Again, this is potentially due to diminished brain endothelial WNT signaling after atRA exposure. Despite alterations in VE-cadherin, Claudin-3, and Claudin-5 expression, BBB integrity was not compromised, indicating that there was sufficient expression of these and other BBB proteins such as ZO-1 to support barrier integrity.

Our analysis of Rdh10 mutants and RA-exposed embryos indicates that the role of RA in BBB maturation is more complex than outlined in recent publications. What accounts for the different outcomes with regard to RA and BBB development? One possibility is that RA is uniquely required for later, postnatal maturation of the BBB that coincides with vessel association of astrocytic endfeet. Mizee et al. (2013) reported that human astrocytes, which they showed to express the RA-biosynthesis protein Raldh, are a possible source of the RA to induce barrier proteins. Another possibility is that the pharmacological concentrations of atRA ( 5 and $10 \mu \mathrm{M}$ ) used in the BBB culture models are activating several pathways in the brain ECs (Mizee et al. 2013; Lippmann et al. 2014; Katt et al. 2016), an effect we have presently tested. RARs bind to atRA with very high affinity (Delescluse et al. 1991), and the physiologic concentration of atRA is thought to be $\sim 25 \mathrm{nM}$ within the developing mouse embryo (Mic et al. 2003). Pharmacological concentrations of atRA, however, can activate RXRs due to the isomerization of atRA to 9cRA, which is the known, high-affinity ligand for RXRs (Urbach and Rando 1994). Given that RAR activation requires concentrations of atRA in the nanomolar range, it is possible that the pharmacological concentrations of atRA used in these studies are activating RXR-mediated signaling, and this contributes to increased expression of BBB proteins and acquisition of barrier properties observed in the published culture studies.

Our studies show that high, pharmacological concentrations of atRA stimulate BBB gene expression in bEnd.3 cells, an effect that has also been observed in human brain ECs (Mizee et al. 2013) and iPSC-derived brain ECs (Lippmann et al. 2014; Katt et al. 2016). We also found that BBB gene expression can be induced with pharmacological doses of 9cRA, possibly through RXR signaling; 


\section{A Retinoic acid is not required for or sufficient to induce BBB protein expression during mouse brain vascular development}

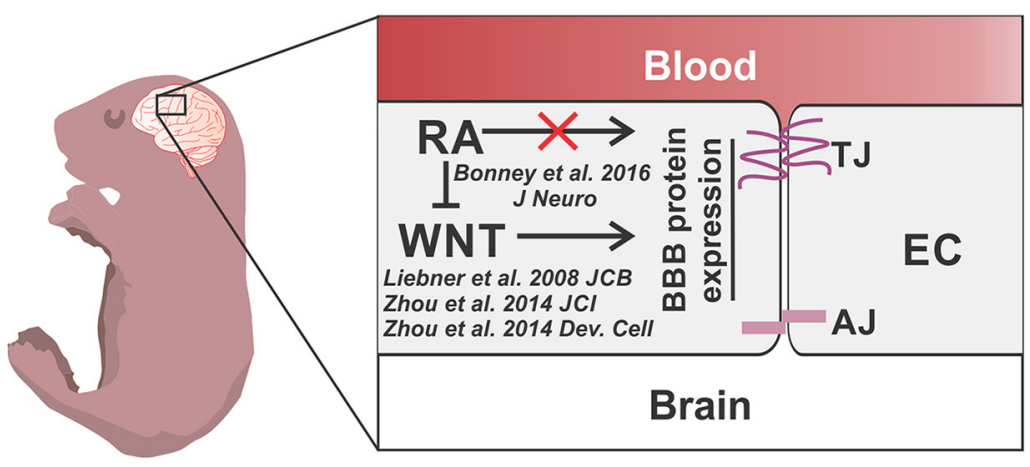

\section{B Pharmacological concentrations of retinoic acid induce BBB protein expression in vitro through RXR-LXR signaling}

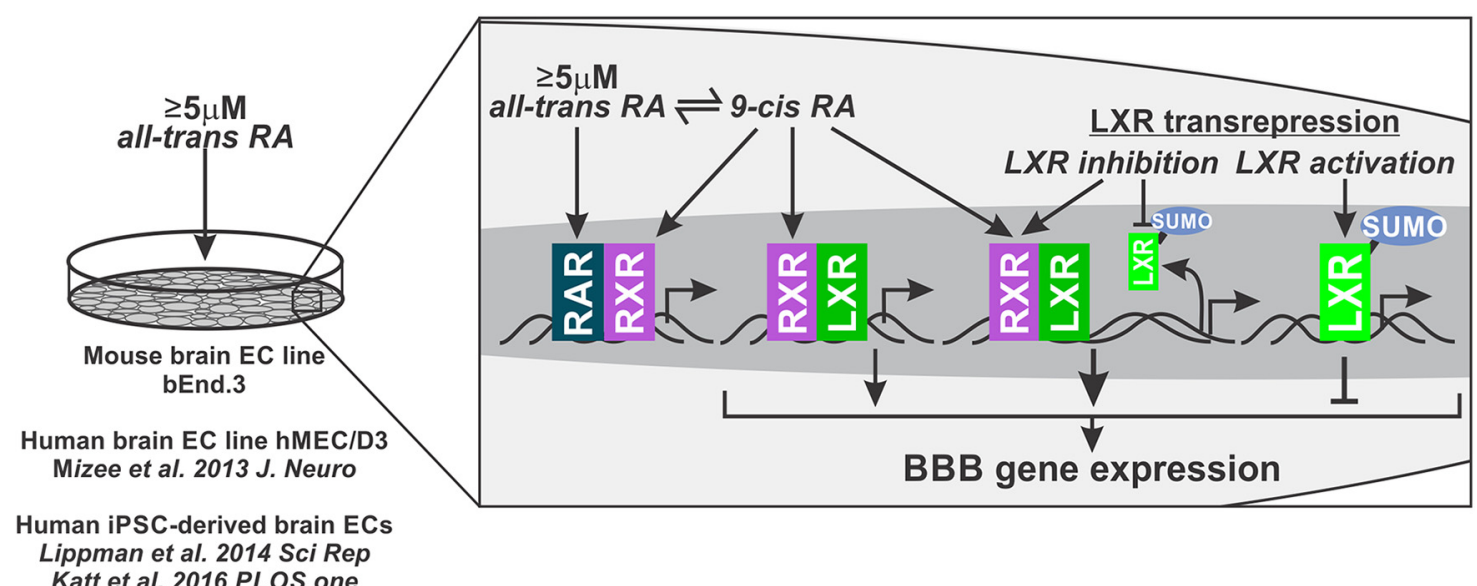

Figure 6. A, Our studies using RA-deficient embryos (Rdh10 mutants) and atRA-exposed embryos revealed that RA is not required for or capable of enhancing BBB protein expression within the developing brain vasculature. Conversely, multiple studies show that endothelial WNT signaling is required for BBB protein expression by the developing brain vasculature (Liebner et al. 2008; Zhou and Nathans 2014; Zhou et al. 2014). A recent study also showed that RA inhibits endothelial WNT signaling, further supporting that RA does not function to promote BBB development (Bonney et al. 2016). B, Recent studies have used pharmacological doses of atRA to induce BBB gene expression in human brain ECs (Mizee et al. 2013) and iPSC-derived brain ECs (Katt et al. 2016; Lippmann et al. 2014). Here we show the same effect occurs in the murine brain endothelioma cell line, b.End 3 cells. Pharmacological concentrations of atRA, which activates RAR/RXR signaling, can also isomerize to 9cRA and activate the LXR/RXR signaling pathway. We also show that 9cRA is capable of inducing BBB gene expression and LXR/RXR signaling in the b.End3 cells. In our studies to identify how LXRs are involved in BBB gene regulation, we found that activation of LXR signaling using T0901317 results in the down-regulation of BBB genes. This effect may act through LXR transrepression in which LXR monomers become SUMOylated and recruit transcriptional corepressors to the promoter of BBB genes. Inhibition of LXR signaling with GSK-2033 reduces LXR SUMOylation and may relieve the LXR transrepression of BBB genes, which enhances the LXR/RXR-mediated up-regulation of BBB genes. Additionally, GSK-2033 can induce RXR transcriptional activity, thus potentially promoting the expression of BBB genes (Griffett and Burris 2016).

specifically, we found activation of LXR/RXR signaling with pharmacological doses of atRA and 9cRA. Our intended use of GSK-2033 was to inhibit LXR activity to test the idea that LXR/RXR signaling activation with $5 \mu \mathrm{M} R A$ was important for BBB gene expression. Just recently, however, GSK-2033 was shown to induce RXR, but not RAR, transcriptional activity (Griffett and Burris 2016). Therefore, our data showing enhanced BBB gene expression with $5 \mu \mathrm{M}$ atRA and GSK-2033, a potential RXR activator, further supports the idea that RXR signaling is involved in $\mathrm{BBB}$ gene regulation in these in vitro BBB models. However GSK-2033 alone did not induce BBB gene expression, and this could be due to disparate mechanisms of RXR activation with 9cRA, atRA, and GSK-2033. Although microarray studies show enrichment of RXR signaling in brain ECs (Daneman et al. 2010), studying the role of RXR signaling in endothelial cells and BBB properties could prove difficult given the many signaling pathways RXRs are involved in and the potential for compensation from the various RXR isoforms. It is important to note, however, that pharmacological levels of RA are a useful method to enhance barrier properties in an 
iPSC-derived BBB model (Lippmann et al. 2014; Katt et al. 2016). This type of model has the potential to be valuable for study of drug delivery and disease. However, a more thorough understanding of the effects of high concentrations of RA and the signaling pathways involved will allow for further optimization of these BBB cell culture models. The bEnd. 3 cells are an attractive cell line to model the BBB in vitro; however, their BBB properties (permeability and $\mathrm{TJ}$ expression) seem to weaken with increasing passage number (Brown et al. 2007), which is a limitation that needs to be taken in consideration when using these cells for such assays.

LXRs are activated by endogenous cholesterol metabolites such as oxysterols and sterols, which are known to up-regulate the ATP-binding cassette $(A B C)$ transporters in brain endothelial cells. These transporters regulate cholesterol transportation and the flux of waste products out of the brain (de Wit et al. 2016). In vivo injury studies have shown that activation of LXR signaling using T0901317 attenuated BBB leakage in mouse models of middle cerebral artery occlusion (EIAli and Hermann 2011) and experimental intracerebral hemorrhage (Wu et al. 2016). However, this could be due to a recent phenomenon identified in innate immune cells, such as macrophages, called LXR transrepression. Upon LXR activation, LXR monomers become SUMOylated and inhibit NF- $\kappa$ B-, AP1-, and STAT1-mediated inflammatory responses by recruiting transcriptional repressors (Jakobsson et al. 2012). Activation of LXRs in these brain injury models could suppress a widespread inflammatory response, thus limiting BBB breakdown. Although it is unclear how LXRs function specifically in endothelial cells, our experiments with the LXR agonist, T0901317, suggest a similar mechanism whereby endothelial LXR may function independently of RXR to suppress BBB gene expression. This is potentially mediated by LXR transrepression, in which activation of LXRs increases the pool of SUMOylated LXR monomers and directly suppresses BBB gene expression (Fig. 6B). In addition, inhibition of LXR activity with GSK2033 may relieve LXR-mediated suppression, therefore allowing for enhanced BBB gene expression by the pharmacological RA treatments through LXR/RXR transcriptional activity (Fig. 6B). Activation of LXR signaling in human umbilical vein endothelial cells (HUVECs) with various oxysterols increased endothelial barrier permeability (Hennig and Boissonneault 1987). This supports our findings that activation of endothelial LXRs may reduce barrier permeability, but it is not clear whether this effect was mediated by LXR transrepression. Later work on HUVECs and human aorta endothelial cells revealed some disparity depending on the mechanism of LXR activation. Oxysterols induced a pro-inflammatory response in endothelial cells (up-regulation of I-CAM and V-CAM; Lemaire et al. 1998); however, treatment with T0901317 attenuated the expression of the immune adhesion molecules I-CAM, $\mathrm{V}-\mathrm{CAM}$, and E-selection in lipopolysaccharide-induced inflammatory states (Morello et al. 2009). Although the role of endothelial LXRs in barrier dysfunction and immune adhesion/infiltration is unclear, these are hallmark events of many neurologic diseases and therefore understanding their role is crucial.

\section{References}

Ashique AM, May SR, Kane MA, Folias AE, Phamluong K, Choe Y, Napoli JL, Peterson AS (2012) Morphological defects in a novel Rdh10 mutant that has reduced retinoic acid biosynthesis and signaling. Genesis 50:415-423. CrossRef

Bauer H-C, Krizbai IA, Bauer H, Traweger A (2014) "You Shall Not Pass"-tight junctions of the blood brain barrier. Front Neurosci 8:392. CrossRef Medline

Bonney S, Harrison-Uy S, Mishra S, MacPherson AM, Choe Y, Li D, Jaminet CS, Fruttiger M, Pleasure SJ, Siegenthaler JA (2016) Diverse functions of retinoic acid in brain vascular development. $J$ Neurosci 36:7786-7801. CrossRef

Brown RC, Morris AP, O'Neil RG (2007) Tight junction protein expression and barrier properties of immortalized mouse brain microvessel endothelial cells. Brain Res 1130:17-30. CrossRef Medline

Daneman R, Zhou L, Agalliu D, Cahoy JD, Kaushal A, Barres BA (2010) The mouse blood-brain barrier transcriptome: a new resource for understanding the development and function of brain endothelial cells. PLoS ONE 5:e13741. CrossRef

de Wit NM, Vanmol J, Kamermans A, Hendriks JJA, de Vries HE (2016) Inflammation at the blood-brain barrier: the role of liver $X$ receptors. Neurobiol Dis pii: S0969-9961(16)30231-5.

Delescluse C, Cavey MT, Martin B, Bernard BA, Reichert U, Maignan J, Darmon M, Shroot B (1991) Selective high affinity retinoic acid receptor alpha or beta-gamma ligands. Mol Pharmacol 40:556562. Medline

EIAli A, Hermann DM (2011) Liver X receptor activation enhances blood-brain barrier integrity in the ischemic brain and increases the abundance of ATP-binding cassette transporters ABCB1 and ABCC1 on brain capillary cells. Brain Pathol (Zurich) 22:175-187. CrossRef

Engelhardt B, Liebner S (2014) Novel insights into the development and maintenance of the blood-brain barrier. Cell Tissue Res 355: 687-699.

Griffett K, Burris TP (2016) Promiscuous activity of the LXR antagonist GSK2033 in a mouse model of fatty liver disease. Biochem Biophys Res Commun 479:424-428. CrossRef

Hawkins BT, Davis TP (2005) The blood-brain barrier/neurovascular unit in health and disease. Pharmacol Rev 57:173-185. CrossRef Medline

Hennig B, Boissonneault GA (1987) Cholestan-3 beta, 5 alpha, 6 betatriol decreases barrier function of cultured endothelial cell monolayers. Atherosclerosis 68:255-261. Medline

Jakobsson T, Treuter E, Gustafsson J-Å, Steffensen KR (2012) Liver $X$ receptor biology and pharmacology: new pathways, challenges and opportunities. Trends Pharmacol Sci 33:394-404. CrossRef Medline

Katt ME, Xu ZS, Gerecht S, Searson PC (2016) Human brain microvascular endothelial cells derived from the BC1 iPS cell line exhibit a blood-brain barrier phenotype. PloS One 11:e0152105. CrossRef

Lemaire S, Lizard G, Monier S, Miguet C, Gueldry S, Volot F, Gambert P, Néel D (1998) Different patterns of IL-1 $\beta$ secretion, adhesion molecule expression and apoptosis induction in human endothelial cells treated with $7 \alpha^{-}, 7 \beta$-hydroxycholesterol, or 7-ketocholesterol. FEBS Lett 440:434-439. Medline

Liebner S, Corada M, Bangsow T, Babbage J, Taddei A, Czupalla CJ, Reis M, Felici A, Wolburg H, Fruttiger M, Taketo MM, von Melchner H, Plate K, Gerhardt H, Dejana E (2008) Wnt/ $\beta$-catenin signaling controls development of the blood-brain barrier. J Cell Biol 183: 409-417. CrossRef Medline

Lippmann ES, Al-Ahmad A, Azarin SM, Palecek SP, Shusta EV (2014) A retinoic acid-enhanced, multicellular human blood-brain barrier model derived from stem cell sources. Sci Rep 4:4160. CrossRef 
Mic FA, Molotkov A, Benbrook DM, Duester G (2003) Retinoid activation of retinoic acid receptor but not retinoid $X$ receptor is sufficient to rescue lethal defect in retinoic acid synthesis. Proc Natl Acad Sci U S A 100:7135-7140. CrossRef Medline

Mizee MR, Wooldrik D, Lakeman KA, van het Hof B, Drexhage JA, Geerts D, Bugiani M, Aronica E, Mebius RE, Prat A, de Vries HE, Reijerkerk A (2013) Retinoic acid induces blood-brain barrier development. J Neurosci 33:1660-1671. CrossRef Medline

Morello F, Saglio E, Noghero A, Schiavone D, Williams T, Verhovez A, Bussolino F, Veglio F, Mulatero P (2009) LXR-activating oxysterols induce the expression of inflammatory markers in endothelial cells through LXR-independent mechanisms. Atherosclerosis 207:3844. CrossRef Medline

Napoli JL, Posch KP, Fiorella PD, Boerman MH (1991) Physiological occurrence, biosynthesis and metabolism of retinoic acid: evidence for roles of cellular retinol-binding protein (CRBP) and cellular retinoic acid-binding protein (CRABP) in the pathway of retinoic acid homeostasis. Biomed Pharmacother 45:131-143. CrossRef

Obermeier B, Daneman R, Ransohoff RM (2013) Development, maintenance and disruption of the blood-brain barrier. Nat Med 19: 1584-1596. CrossRef

Siegenthaler JA, Sohet F, Daneman R (2013) 'Sealing off the CNS': cellular and molecular regulation of blood-brain barriergenesis. Curr Opin Neurobiol 23:1057-1064. CrossRef Medline
Szanto A, Narkar V, Shen Q, Uray IP, Davies PJA, Nagy L (2004) Retinoid $X$ receptors: $X$-ploring their (patho)physiological functions. Cell Death Differ 11(Suppl 2):126-143. CrossRef

Tanoury AZ, Piskunov A, Rochette-Egly C (2013) Vitamin A and retinoid signaling: genomic and nongenomic effects: Thematic Review Series: Fat-Soluble Vitamins: Vitamin A. J Lipid Res 54: 1761-1775. CrossRef

Urbach J, Rando RR (1994) Isomerization of all-trans-retinoic acid to 9-cis-retinoic acid. Biochem J 299:459-465. CrossRef

Watanabe T, Dohgu S, Takata F, Nishioku T, Nakashima A, Futagami K, Yamauchi A, Kataoka Y (2013) Paracellular barrier and tight junction protein expression in the immortalized brain endothelial cell lines bEND.3, bEND.5 and mouse brain endothelial cell 4. Biol Pharm Bull 36:492-495. Medline

Wu C-H, Chen C-C, Lai C-Y, Hung T-H, Lin C-C, Chao M, Chen S-F (2016) Treatment with TO901317, a synthetic liver $X$ receptor agonist, reduces brain damage and attenuates neuroinflammation in experimental intracerebral hemorrhage. J Neuroinflamm 13:62. CrossRef Medline

Zhou Y, Nathans J (2014) Gpr124 controls CNS angiogenesis and blood-brain barrier integrity by promoting ligand-specific canonical wnt signaling. Dev Cell 31:248-256. CrossRef Medline

Zhou Y, Wang Y, Tischfield M, Williams J, Smallwood PM, Rattner A, Taketo MM, Nathans J (2014) Canonical WNT signaling components in vascular development and barrier formation. $J$ Clin Invest 124:3825-3846. CrossRef Medline 